\title{
Reconstruction of Wireless UWB Pulses by Exponential Sampling Filter
}

\author{
Juuso T. Olkkonen ${ }^{1}$, Hannu Olkkonen ${ }^{2}$ \\ ${ }^{1}$ VTT Technical Research Centre of Finland, Espoo, Finland \\ ${ }^{2}$ Department of Physics and Mathematics, University of Eastern Finland, Kuopio, Finland \\ E-mail:juuso.olkkonen@vtt.fi,hannu.olkkonen@uef.fi \\ Received November 16, 2009; revised December 25, 2009; accepted January 20, 2010
}

\begin{abstract}
Measurement and reconstruction of wireless pulses is an important scheme in wireless ultra wide band (UWB) technology. In contrary to the band-limited analog signals, which can be recovered from evenly spaced samples, the reconstruction of the UWB pulses is a more demanding task. In this work we describe an exponential sampling filter (ESF) for measurement and reconstruction of UWB pulses. The ESF is constructed from parallel filters, which has exponentially descending impulse response. A pole cancellation filter was used to extract the amplitudes and time locations of the UWB pulses from sequentially measured samples of the ESF output. We show that the amplitudes and time locations of $p$ sequential UWB pulses can be recovered from the measurement of at least $2 p$ samples from the ESF output. For perfect reconstruction the number of parallel filters in ESP should be $2 p$. We study the robustness of the method against noise and discuss the applications of the method.
\end{abstract}

Keywords: Wireless Sensor Networks, UWB, Network Security, Finite Rate of Innovation

\section{Introduction}

The measurement and reconstruction of some classes of signals containing discontinuities such as impulses and edges is difficult $[1,2]$. Sampling methods are historically relied on Shannon's theorem [3]. The perfect reconstruction (PR) of the continuous signal from the sampled version requires that the signal is band limited, i.e., its frequency spectrum has a maximum frequency $f_{M}$. The PR is possible only if the sampling frequency $f_{s} \geq 2 f_{M}$. For example, signals in optical devices and radiation detectors are not band limited and classical sampling approaches are not relevant for extracting the information.

Sampling scheme with finite rate of innovation (FRI) $[4,5]$ has recently got vast interest in signal processing society, since the FRI methods are not restricted to the recovery of the band-limited signals. The key idea in FRI methods is that the signal (e.g., the Dirac impulse stream) is measured with a sampling filter, which is constructed using analog circuits. The output of the sampling filter is measured and the original signal is reconstructed from the discrete samples. Excellent articles and reviews consider the FRI sampling and reconstruction techniques
[4-9] and many feasible applications have been published. However, the experimental work on the verification of the underlying theoretical considerations is lacking, especially the effect of noise on the reconstruction accuracy.

The information in wireless ultra wideband (UWB) devices is usually carried out by monocycle Gaussian pulses. In year 2002 the FCC restricted the allowed frequency band between 3.1-10.6 GHz for unlicensed UWB transmission [10]. The monocycle Gaussian pulse stream does not strictly meet this constraint and other pulse shapes have been introduced, e.g., the family of the orthogonal UWB pulse waveforms [11]. In low-range wireless UWB communication devices, which transmit sequential pulses, the information is coded to the time locations of the pulses. The UWB pulse generators are relatively easy to implement in VLSI [12]. The pulse stream is designed so that its power spectral density coincides with the FCC criteria.

In this work we study the FRI-like method aimed at sampling and reconstruction of the UWB pulses. As a sampling device we apply an exponential sampling filter (ESF), whose output is measured sequentially. We study the robustness of the method against noise and discuss the applications of the method. 


\section{Theoretical Considerations}

\subsection{Sampling of the UWB Pulse Train}

We consider the UWB pulse train, which is approximated by

$$
I(t)=\sum_{i=1}^{p} A_{i} \delta\left(t-t_{i}\right)
$$

where the Dirac distribution $\delta(x)=1$ for $x=0$ and $\delta(x)=0$ for $x \neq 0 . A_{i}$ denotes the amplitude and $t_{i}$ time location. The pulse train is fed to the exponential sampling filter (ESF) consisting of $N$ parallel RC-filters (Figure 1). The ESF has the causal impulse response

$$
h(t)=\sum_{k=1}^{N} B_{k} \exp [-\alpha k t] \quad t \geq 0
$$

The output signal of the ESF is

$$
\begin{aligned}
& x(t)=I(t) * h(t)=\int_{-\infty}^{\infty} I(\tau) h(t-\tau) d \tau \\
& =\sum_{i=1}^{p} A_{i} \sum_{k=1}^{N} B_{k} \exp \left[-\alpha k\left(t-t_{i}\right)\right] \quad t \geq 0
\end{aligned}
$$

The key idea is to start the measurement of the ESF output at time $t_{0} \geq t_{i}(i=1,2, \ldots, p)$, simultaneously closing the input of the ESF (Figure 1). We define the discrete time variable $n$ as

$$
t=t_{0}+n T(n=0,1,2, \ldots) \Rightarrow n T=t-t_{0}
$$

where $T$ is a sampling period. For the samples of the ESF output $x_{n}=x[n T], n=0,1,2, \ldots$ the following is valid

$$
x_{n}=\sum_{i=1}^{p} A_{i} \sum_{k=1}^{N} B_{k} \exp \left[-\alpha k\left(t_{0}+n T-t_{i}\right)\right]
$$

By rearranging and denoting $\Delta_{i}=t_{o}-t_{i}$

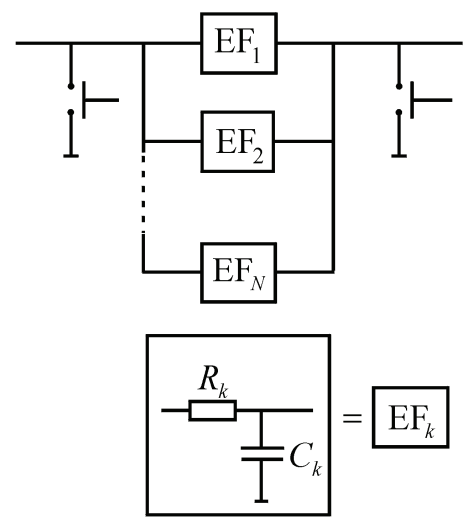

Figure 1. The construction of the ESF from the parallel exponential filters (EF), which are built using RC-circuits. The ESF parameter $\alpha$ is related as $\alpha k T=1 /\left(\mathrm{R}_{\mathrm{k}} \mathrm{C}_{\mathrm{k}}\right)$, where $R_{k}$ and $C_{k}$ are the component values.

$$
x_{n}=\sum_{k=1}^{N} B_{k} \sum_{i=1}^{p} A_{i} e^{-\alpha k \Delta_{i}}\left(e^{-\alpha k T}\right)^{n}=\sum_{k=1}^{N} b_{k} \lambda_{k}^{n}
$$

where

$$
b_{k}=B_{k} \sum_{i=1}^{p} A_{i} e^{-\alpha k \Delta_{i}} \text { and } \lambda_{k}=e^{-\alpha k T}
$$

\subsection{Reconstruction of the Pulse Train}

Our task is to recover the amplitudes and time locations of the pulses from the measured output samples $x_{n}=x[n T], n=0,1,2, \ldots$ We may write (5) in the form of matrix/vector equation

$$
\begin{aligned}
{\left[\begin{array}{c}
x_{0} \\
x_{2} \\
\vdots \\
x_{N-1}
\end{array}\right]=} & {\left[\begin{array}{cccc}
1 & 1 & \cdots & 1 \\
\lambda_{1} & \lambda_{2} & \cdots & \lambda_{N} \\
\vdots & \vdots & \vdots & \vdots \\
\lambda_{1}^{N-1} & \lambda_{2}^{N-1} & \cdots & \lambda_{N}^{N-1}
\end{array}\right]\left[\begin{array}{c}
b_{1} \\
b_{2} \\
\vdots \\
b_{N}
\end{array}\right] } \\
& \Leftrightarrow \mathbf{x}=\lambda \mathbf{b} \Rightarrow \mathbf{b}=\lambda^{-1} \mathbf{x}
\end{aligned}
$$

The Vandermonde matrix structure $\lambda$ in (7) is nonsingular having rank $N$ and the solution of the vector $\mathbf{b}$ requires knowledge of the $N$ measurement values. From (6) we obtain

$$
c_{k}=b_{k} / B_{k}=\sum_{i=1}^{p} A_{i} e^{-\alpha k \Delta_{i}}=\sum_{i=1}^{p} A_{i} r_{i}^{k}
$$

where

$$
r_{i}=e^{-\alpha \Delta_{i}}
$$

The $z$ transform of the $c_{k}$ sequence gives

$$
\begin{aligned}
Z\left\{c_{k}\right\} & =\sum_{k=1}^{N} \sum_{i=1}^{p} A_{i} r_{i}^{k} z^{-k}=\sum_{i=1}^{p} A_{i} \sum_{k=0}^{N-1}\left(r_{i} z^{-1}\right)^{k+1} \\
& =\sum_{i=1}^{p} \frac{A_{i} r_{i} z^{-1}\left(1-r_{i}^{N} z^{-N}\right)}{1-r_{i} z^{-1}}
\end{aligned}
$$

We define the pole cancellation filter (PCF) as

$$
H_{p c}(z)=1+\sum_{n=1}^{p} h_{n} z^{-n}=\prod_{j=1}^{p}\left(1-r_{j} z^{-1}\right)
$$

where $r_{j}$ is defined by (9). Next we consider the product filter $P(z)$

$$
\begin{aligned}
& P(z)=Z\left\{c_{k}\right\} H_{p c}(z) \\
& =\sum_{i=1}^{p} A_{i} r_{i} z^{-1}\left(1-r_{i}^{N} z^{-N}\right) \prod_{\substack{j=1 \\
j \neq i}}^{p}\left(1-r_{j} z^{-1}\right)
\end{aligned}
$$

We may note that the roots of the $P(z)$ equal the roots of the PCF. The impulse response of the $P(z)$ is

$$
p_{n}=\sum_{k=0}^{p} c_{n-k} h_{k}
$$

For solution of the roots of the $P(z)$ we set $p_{n}=0$ for $n \geq 0$. This yields the matrix/vector equation 


$$
\begin{aligned}
{\left[\begin{array}{c}
c_{2 p} \\
c_{2 p-1} \\
\vdots \\
c_{p+1}
\end{array}\right]+\left[\begin{array}{cccc}
c_{2 p-1} & c_{2 p-2} & \cdots & c_{p} \\
c_{2 p-2} & c_{2 p-3} & \cdots & c_{p-1} \\
\vdots & \vdots & \vdots & \vdots \\
c_{p} & c_{p-1} & \cdots & c_{1}
\end{array}\right]\left[\begin{array}{c}
h_{1} \\
h_{2} \\
\vdots \\
h_{p}
\end{array}\right]=0 } \\
\Leftrightarrow \mathbf{c}=-\mathbf{C h} \Rightarrow \mathbf{h}=-\mathbf{C}^{-1} \mathbf{c}
\end{aligned}
$$

We may note that the solution of the $p$ coefficients of the PCF requires the knowledge of the $2 p$ values of the $c_{k}$ sequence. The polynomial $\left[1 h_{1} h_{2} \ldots h_{p}\right]$ has the roots $r_{i}=e^{-\alpha \Delta_{i}}(i=1,2, \ldots, p)$, which give $\Delta_{i}=-\log$ $\left(r_{i}\right) / \alpha$ and the time locations as $t_{i}=t_{0}+\log \left(r_{i}\right) / \alpha$. For solution of the amplitudes we may write (8) in matrix/vector form

$$
\begin{gathered}
{\left[\begin{array}{c}
c_{1} \\
c_{1} \\
\vdots \\
c_{p}
\end{array}\right]=\left[\begin{array}{cccc}
r_{1} & r_{2} & \cdots & r_{p} \\
r_{1}^{2} & r_{2}^{2} & \cdots & r_{p}^{2} \\
\vdots & \vdots & \vdots & \vdots \\
r_{1}^{p} & r_{2}^{p} & \cdots & r_{p}^{p}
\end{array}\right]\left[\begin{array}{c}
A_{1} \\
A_{2} \\
\vdots \\
A_{p}
\end{array}\right]} \\
\Leftrightarrow \mathbf{c}=\mathbf{R} \mathbf{a} \Rightarrow \mathbf{a}=\mathbf{R}^{-1} \mathbf{c}
\end{gathered}
$$

To summarize, the reconstruction of $p$ sequential pulses needs the measurement of at least $2 p$ samples from the ESF output. The impulse response of the ESF must obey (2), where $N=2 p$. It should be pointed out that the solution of the matrix Equations (7) and (15) require the inversion of the Vandermonde matrix, which can be obtained by an analytical formula [13]. The inversion method yields more stable results compared with the general matrix inversion.

\subsection{Reduction of Noise}

In practical measurements noise arising in electronic circuits interferes the results. We apply the SVD based subspace method for reducing the noise in measurement signal. Let us construct the Hankel matrix containing the measurement values $x_{n}=x[n T],(n=0, \ldots, M \geq 2 p)$

$$
\mathbf{H}=\left[\begin{array}{cccc}
x_{0} & x_{1} & \cdots & x_{M / 2} \\
x_{1} & x_{2} & \cdots & x_{M / 2+1} \\
\vdots & \vdots & \vdots & \vdots \\
x_{M / 2} & x_{M / 2+1} & \cdots & x_{M}
\end{array}\right]
$$

where the antidiagonal elements are identical. The singular value decomposition (SVD) of the matrix $\mathbf{H}$ is

$$
\mathbf{H}=\mathbf{U} \boldsymbol{\Sigma} \mathbf{V}^{\mathbf{T}}
$$

where $\mathbf{U}$ and $\mathbf{V}$ are unitary matrices. $\boldsymbol{\Sigma}$ is a diagonal matrix consisting of the singular values in descending order. The decomposition (17) can be separated as

$$
\begin{aligned}
\mathbf{H} & =\left[\begin{array}{ll}
\mathbf{U}_{\mathrm{s}} & \mathbf{U}_{\mathrm{n}}
\end{array}\right]\left[\begin{array}{rr}
\boldsymbol{\Sigma}_{\mathrm{s}} & 0 \\
0 & \Sigma_{\mathrm{n}}
\end{array}\right]\left[\begin{array}{ll}
V_{\mathrm{s}} & \mathbf{V}_{\mathrm{n}}
\end{array}\right]^{\mathrm{T}} \\
& =\mathbf{U}_{\mathrm{s}} \boldsymbol{\Sigma}_{\mathrm{s}} \mathbf{V}_{\mathrm{s}}^{\mathrm{T}}+\mathbf{U}_{\mathrm{n}} \boldsymbol{\Sigma}_{\mathrm{n}} \mathbf{V}_{\mathrm{n}}^{\mathrm{T}}=\mathbf{H}_{\mathrm{s}}+\mathbf{H}_{\mathrm{n}}
\end{aligned}
$$

where $\boldsymbol{\Sigma}_{\mathbf{n}}$ contains the smallest singular values. $\mathbf{H}_{\mathbf{n}}$ matrix belongs to noise subspace. The matrix $\mathbf{H}_{\mathbf{s}}$ is then related to the noise free signal subspace [14]. The signal matrix $\mathbf{H}_{\mathbf{s}}$ is not precisely Hankel matrix, but some variation occurs in the antidiagonal elements. We reconstructed the noise free Hankel matrix by replacing the antidiagonal elements by their mean values. This enables the computation of the noise cancelled $x_{n}$ $(n=0,1, \ldots, M)$ sequence. The dimension of the signal subspace is $N$, i.e., the number of parallel RC-circuits in ESF. It should be pointed out that the Hankel matrix (16) must be a full matrix. Therefore $M$ should be even.

\section{Experimental}

Extensive simulations were performed to validate the theoretical results. The $\alpha T$ parameter in (5) varied between $0.1-0.3$. The number of the impulses in one burst varied between 3-7 and the number $N$ in ESF (2) in the range 3-15. The amplitudes of the pulses were randomly distributed between the limits 0.1-0.9. The simulations warranted the condition that for the recovery of $p$ pulses at least $2 p$ samples are needed in the descending part of the ESF output. A typical simulation study is illustrated in Figure 2. In every case the ESF method recovered the amplitudes and the time locations of the pulses with a machine precision.

A prototype ESF was constructed with the aid of six parallel RC-circuits (Figure 1). The output was measured by a 16 bit analog-to-digital converter (ADC), which was equipped with a sample and hold amplifier $(\mathrm{S} / \mathrm{H})$. The input of the ESF was closed using the analog CMOS switch. The ESF was reset by grounding the output by an analog CMOS switch. Using a commercial UWB pulse generator two sequential pulses were fed to the input of the ESF and the descending part was measured with a $40 \mathrm{MHz}$ sampling frequency. Due to the noise interference in practical measurements the use of the SVD based noise suppression method was essential. The prototype ESF recovered the amplitudes and the appearance times with a good accuracy. The mean error (standard deviation divided by the mean value) in amplitudes was $2.7 \%$ and in time locations $0.2 \%$.

\section{Discussion}

The present work proposes a new approach for measuring of the impulse train using the ESF. The ESF yields exponentially descending pulses, which are sampled sequentially. In the beginning of the measurement period the input is closed and after measurement period the ESF is reset. We showed that using $2 p$ parallel $\mathrm{RC}$ filters it is possible to reconstruct $p$ impulses. The required number 


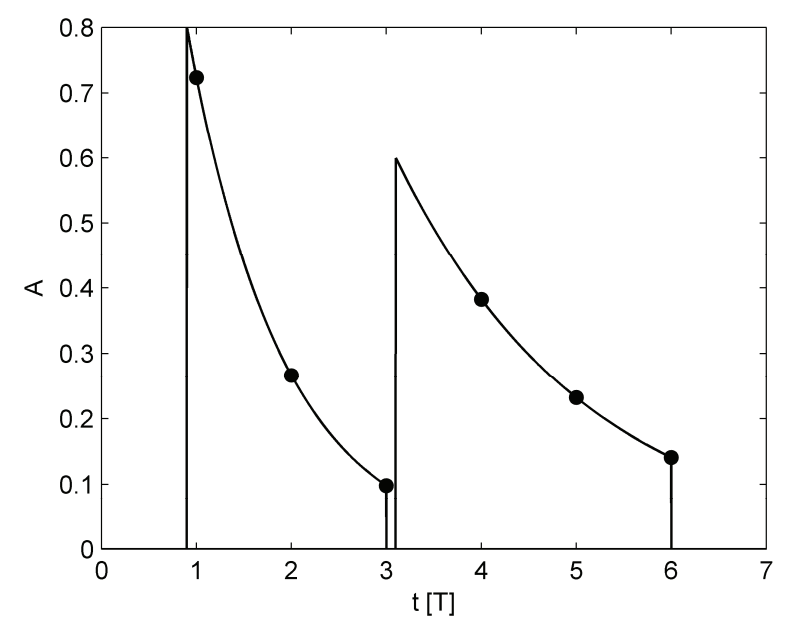

Figure 2. The minimum time interval $d t_{\mathrm{i}}=t_{i}-t_{i-1}$ must be $d t_{i}>2 T$ for perfect reconstruction using ESF with two parallel RC circuits and three sequential samples per impulse. The dashed circles denote the measurement values and the solid line reconstructed ESF output via Equation (3).

of the samples was proved to be at least $2 p$. The simulations using noise free signal indicated an accurate and precise reconstruction property, in practice the ESF method showed a high sensitivity to the noise. To obtain a tolerable reconstruction error, the ESF circuit requires Faraday gage-type shielding and careful consideration of grounding and signal cables. The experimental verification of the effect of different types of noise sources (50 Hz + harmonics pick-up, 1/f-noise and random noise) needs further study.

In this work the noise was cancelled using the SVD based subspace method (16-18), which eliminates the noise interference if the number of samples exceeds $2 p$. The key idea is to compute the noise free signal as a mean of the antidiagonal elements in the signal subspace matrix $\mathbf{H}_{\mathbf{s}}$. The error in the noise cancelled sequence $x_{n}(n=0,1, \ldots, M)$ attains a minimum in the middle of the sequence, where $M$ data points are used for computation of the mean value. Evidently the higher error in both ends of the sequence is due to the lower number of points.

The ESF method has a close relationship with the FRI methods, which are based on the sequential measurement of the output of the analog circuit network [5]. The reconstruction properties of the Gaussian, sinc-type and triangle-wave sampling filters have been described [4-6, 8 ], but to the best of the authors knowledge, ESF-type circuits have not been previously used for the sampling and recovery of the UWB pulse sequences. A clear difference between the FRI sampling filters and the ESF comes from causality. An exponential impulse response $\exp \left[-\alpha k\left(t-t_{i}\right)\right]$ is causal and can be separated in (5) only if $t \geq t_{i}$. On the contrary, FRI sampling filters are not causal and this restriction is not needed in the deduction of the reconstruction algorithms. The FRI reconstruction algorithms usually require the solution of two matrix/vector equations, which are Yale-Walker and Vandermonde structures. In our approach three matrix/ vector solutions $(7,14,15)$ are required.

The present ESF measurement scheme can be considered as a general framework for measurement of the UWB pulse amplitudes and time locations. The experiments showed that the reconstruction error is mainly due to the additive random noise affecting on the amplitudes of the pulses. Hence, as an example of the practical construction of the wireless sensor network the ratio of the two sequential UWB pulse amplitudes can be related to the device address. The ratio of the UWB pulse amplitudes is not affected by the attenuation and distance variations. The time difference between the UWB pulses is reconstructed more accurately and it can be used to code the transmitted information.

The ESF method can be applied in many areas of wireless sensor technology and instrumentation. For example, in optical instruments the time difference between laser pulses can be used in testing light transmission in optical fibres. Many other sensors have pulsetype outputs.

\section{Acknowledgements}

This work was supported by the National Technology Agency of Finland (TEKES).

\section{References}

[1] J. Haupt and R. Nowak, "Signal Reconstruction from Noisy Random Projections," IEEE Transactions Information Theory, Vol. 52, No. 9, 2006, pp. 4036-4048.

[2] Y. C. Eldar and M. Unser, "Nonideal Sampling and Interpolation from Noisy Observations in Shift-Invariant Spaces," IEEE Transactions Signal Processing, Vol. 54, No. 7, 2006, pp. 2636-2651.

[3] M. Unser, "Sampling-50 Years after Shannon," Proceedings of IEEE, Vol. 88, No. 4, 2000, pp. 569-587.

[4] P. Marziliano, "Sampling Innovations," Ph.D. Dissertation, Communications laboratory, Lausanne, Switzerland, 2001.

[5] M. Vetterli, P. Marziliano and T. Blu, "Sampling Signals with Finite Rate of Innovation," IEEE Transactions Signal Processing, Vol. 50, No. 6, 2002, pp. 1417-1428.

[6] I. Maravic and M. Vetterli, "Sampling and Reconstruction of Signals with Finite Rate of Innovation in the Presence of Noise," IEEE Transactions Signal Processing, Vol. 53, No. 8, 2005, pp. 2788-2805.

[7] P. Marziliano, M. Vetterli and T. Blu, "Sampling and Exact Reconstruction of Bandlimited Signals with Additive 
Shot Noise," IEEE Transactions Information Theory, Vol. 52, No. 5, 2006, pp. 2230-2233.

[8] P. L. Dragotti, M. Vetterli and T. Blu, "Sampling Moments and Reconstructing Signals of Finite Rate of Innovation: Shannon Meets Strang-Fix," IEEE Transactions Signal Processing, Vol. 55, No. 5, 2007, pp. 1741-1757.

[9] I. Jovanovic and B. Beferull-Lozano, "Oversampled A/D Conversion and Error-Rate Dependence of Nonband Limited Signals with Finite Rate of Innovation," IEEE Transactions Signal Processing, Vol. 54, No. 6, 2006, pp. 2140-2154.

[10] Y. P. Nakache and A. F. Molisch, "Spectral Shaping of UWB Signals for Time-Hopping Impulse Radio," IEEE Journal of Selected Areas of Communications, Vol. 24, No. 4, 2006, pp. 738-744.
[11] B. Parr, B. Cho, K. Wallace and Z. Ding, "A Novel Ultra-Wideband Pulse Design Algorithm," IEEE Communications Letters, Vol. 7, No. 5, 2003, pp. 219-221.

[12] M. Miao and C. Nguyen, "On the Development of an Integrated CMOS-Based UWB Tunable-Pulse Transmit Module," IEEE Transactions Microwave Theory and Techniques, Vol. 54, No. 10, 2006, pp. 3681-3687.

[13] V. E. Neagoe, "Inversion of the Van Der Monde Matrix," IEEE Signal Processing Letters, Vol. 3, No. 4, 1996, pp. 119-120.

[14] E. Biglieri and K. Yao, "Some Properties of Singular Value Decomposition and their Applications to Digital Signal Processing," Signal Processing, Vol. 18, No. 3, 1989, pp. 277-289. 